\title{
The Varying Influences of Positive Emotions on Consumption Motivation
}

\author{
Saran Wanglee \\ Chulalongkorn University \\ E-Mail: swanglee@gmail.com
}

\begin{abstract}
This research investigates how subjective emotional experiences - which should be largely irrelevant to judgment and choices - impact consumers' reward-seeking behaviors. Extant theories of affective influences on consumption motivation have taken a valence-based approach, contrasting the effects of positive versus negative states of emotion. These approaches have not specified if and when distinct emotions of the same valence have different effects on motivation. This research not only demonstrates that positive emotions have a wide range of influences on motivation, but also how frequent exposure to distinct and unrelated positive emotions systematically affects reward-seeking behaviors.
\end{abstract}

Keywords: Motivation, Emotions, Reward Seeking, Consumption

\section{INTRODUCTION}

Historically, decision-making was viewed as a cognitive process - a matter of making a choice with the intention of maximizing the positive consequences (Loewenstein \& Lerner, 2003). In psychologists' line of thinking, it is analogous to the theory of utility maximization espoused by economists, whereby people are assumed to be rational. Yet countless research studies have shown that human behavior fails to comply with the assumption of rationality.

Humans are so often irrational because our feelings frequently interfere with our choices. A summary of such research can be found in Dan Ariely's books, Predictably Irrational (Ariely \& Jones, 2008), and The Upside of Irrationality (Ariely, 2010). The past two decades have witnessed growing interest in research into the role emotions play in judgment and choice (for review, see Han, Lerner \& Keltner, 2007). For example, Johnson and Tversky (1983) demonstrated that positive emotions lead 
people to make more optimistic assessments of their choices than negative emotions. Conversely, negative emotions have been shown to lead people to make more pessimistic assessments of their choices than positive emotions (Wright \& Bower, 1992). More recently, Custers and Aarts (2005), Aarts, Custers \& Marien (2008), and Baumeister et al. (2007) further demonstrated that a positive emotional state is an important aspect of an individual's goal representation; the state motivates people to behave in ways that that assist in reaching their goals. Once a goal has been achieved, this positive feeling then dissipates (Atkinson \& Birch, 1970). These studies investigate what Loewenstein and Lerner (2003) termed "integral emotion": emotion which encompasses influences of subjective experiences that are normatively relevant to present judgments and choices.

On the other hand, emotions may also be "incidental." Incidental emotions are the subjective emotional experiences that should be normatively irrelevant to present judgment and choices (for review, see Forgas \& Locke, 2005; Lerner \& Keltner, 2000). For example, emotions produced by listening to music, experiencing bad weather, or reliving stressful events have been shown to influence judgments on unrelated topics and objects (Bodenhausen, Kramer \& Süsser, 1994; Forgas \& Bower, 1988; Schwarz \& Clore, 1983). Such incidental carryover occurs even when decisionmakers are unaware of such influences, and also when concrete economic outcomes are at stake (Lerner, Small \& Loewenstein, 2004).

However, the impact of emotions on behaviors extends beyond the distinction between positive and negative emotions. Recent research has demonstrated the importance of examining specific emotions in addition to global positive and negative feelings (Bodenhausen et al., 1994; Lerner, Han \& Keltner, 2007a). For example, in their investigation of incidental negative emotions on the endowment effect ${ }^{1}$, Lerner et al. (2004) showed that the difference between the selling and buying price of a specific item was not significant when the subject was primed with disgust (no endowment effect), while they documented a reverse endowment effect (buying price exceeded selling price) when the subject was primed with sadness. Paradoxically, both disgust and sadness are undeniably negative emotions, and yet they produce systematically different outcomes. This finding would not have been documented had emotions been parsed as simply positive or negative.

This brief review has shown that the role of emotion extends beyond the conventional "end state" feeling resulting from performing some relevant actions. It demonstrates that each positive emotion has motivational properties (Custers \& Aarts,

\footnotetext{
${ }^{1}$ Endowment effect: the tendency for selling prices to exceed buying prices for the same object
} 
2005; Aarts, Custers \& Marien, 2008) that may drive subsequent unrelated behaviors differently (Lerner, Han \& Keltner, 2007b). To this end, the proposed question is:

Could one positive emotion serve to satiate an activated motivational state, while another positive emotion serves to bolster a motivational state to pursue a goal?

\section{CONCEPTUAL DEVELOPMENT}

This inquiry has been partially answered by research on the relationship between product sampling and consumption motivation (Wadhwa, Shiv \& Nowlis, 2008). These researchers first demonstrated that subjects who sampled chocolate in the first phase subsequently and unconsciously indulged in more soft drinks and snacks in the second phase ${ }^{2}$ than did those subjects who had not sampled the chocolate. This effect, termed reverse alliesthesia, was eliminated for those subjects who received $\$ 1$ after eating the chocolate (i.e., subjects who consumed the same amount of soft drinks and snacks as those who did not eat the chocolate). The authors claimed that receiving the $\$ 1$ was a reward (an induced positive emotion) that satiated the motivation induced from sampling. In this paper, the conditions under which subjects receiving a $\$ 1$ reward end up consuming more soft drinks and snacks are investigated. In other words, I will attempt to determine when a given stimulus serves as a motivator and when it serves as an end state in and of itself.

In order to better understand this specific inquiry, a review of the literature associated with reverse alliesthesia is first presented, highlighting evidence in support of its manifestation. I then elaborate on some of the problems related to that body of work.

\section{The Phenomenon of Reverse Alliesthesia}

Common intuition suggests that food and beverage sampling should make consumers less hungry or thirsty, and thus reduce their subsequent consumption behavior. However, prior work on alliesthesia has shown that an increase in a physiological drive state (e.g., thirst, hunger) enhances the impact (i.e., incentive value) of a consumption cue such as water or food (Cabanac, 1979). More recent research has been recast in a manner suggesting that alliesthesia may occur in the reverse order as well. That is, just as drive states affect the incentive value of a relevant reward stimulus, encountering a stimulus that is high in incentive value could also intensify motivational states and thus enhance the desire to engage in subsequent consumption-related behaviors (Berridge, 2000, 2001). This phenomenon is referred

\footnotetext{
${ }^{2}$ First and second phase separated in topic, time, location
} 
to as reverse alliesthesia (Wadhwa et al., 2008). For example, reverse alliesthesia suggests that drinking a small quantity of fruit punch will enhance thirst and thereby increase the desire for more beverages.

Research in support of reverse alliesthesia suggests that its effects operate at three levels of specificity: specific to the consumption cue (Cornell, Rodin \& Weingarten, 1989), specific to a drive state (Berridge, 2001; Toates, 1986), and general to a broader motivational state (Gray, 1987; Kambouropoulos \& Staiger, 2001). A summary of the process and effects is provided in Figure 1.

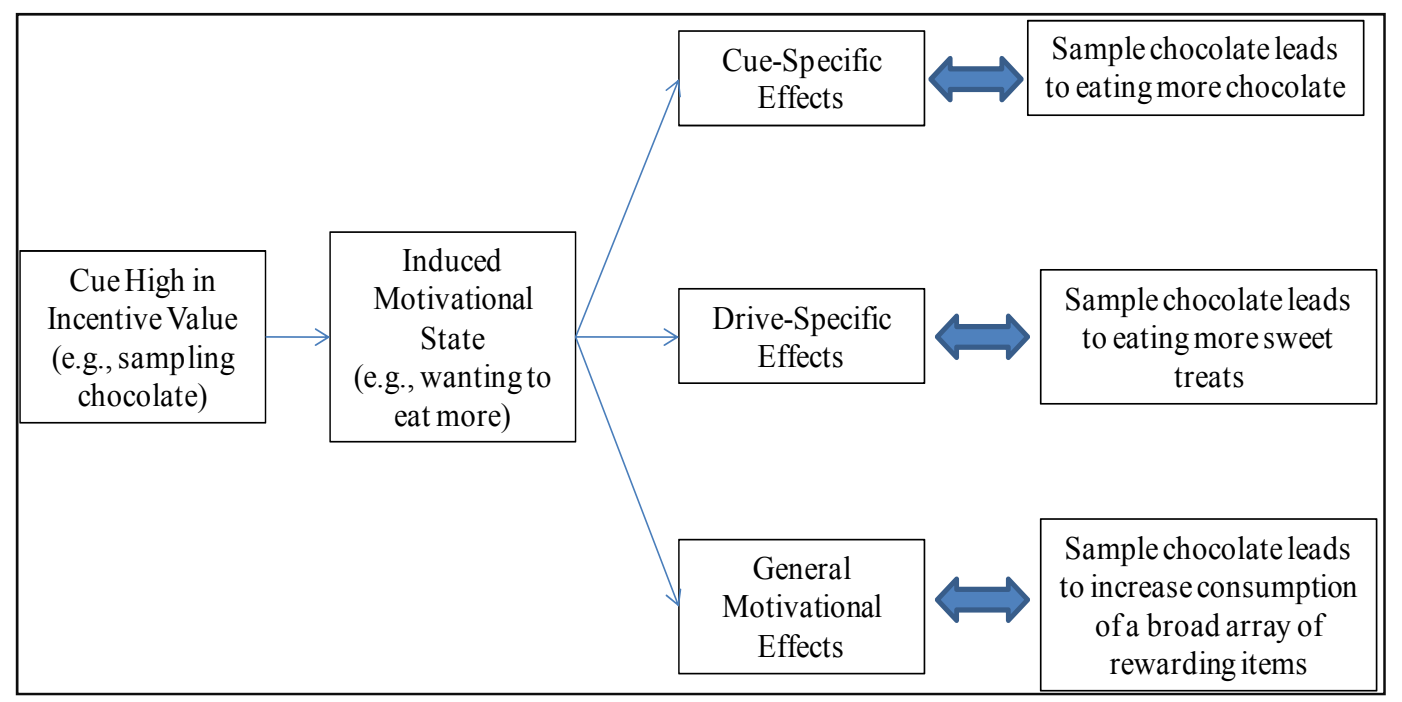

Figure 1 Process and Effects of Reverse Alliesthesia

The above constructs at cue-specific, drive-specific, and general motivational level have been experimentally validated by Wadhwa et al. (2008). Specifically, Experiment 1 demonstrated that sampling a drink high in incentive value (a delicious fruit punch) increased subjects' desire to consume more soda later - a prediction arising from reverse alliesthesia - rather than decreasing the desire to drink more (a prediction arising from common intuition). In Experiment 2, researchers showed that giving subjects $\$ 1$ after their first drink satiated the induced motivational state when subjects were presented with a subsequent consumption task; thus, the cash reward eliminated the drive-specific effects found in Experiment 1 (Figure 2). The authors argued that the $\$ 1$ reward was a manifestation of the general motivational hypothesis, which then eliminated the induced motivational state to engage in drive-specific consumptions. 


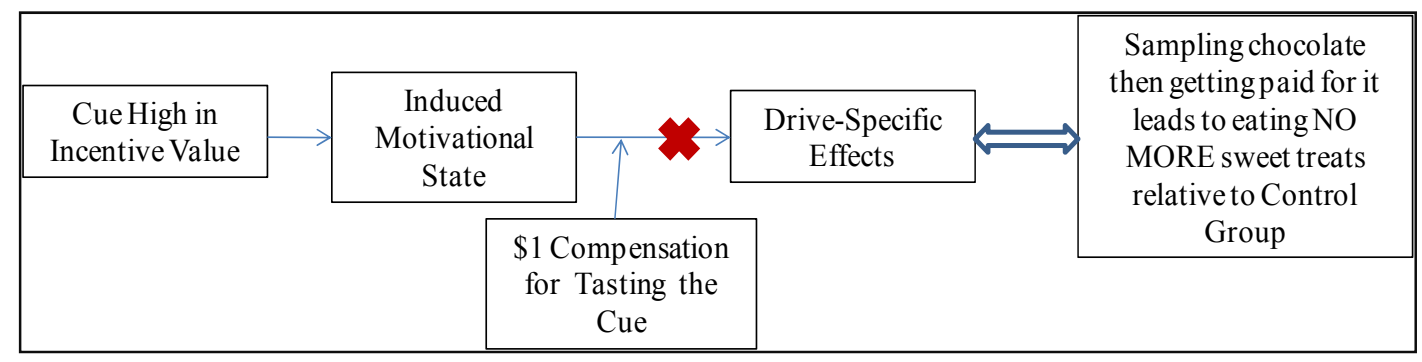

Figure 2 Satiating Motivation With A High-Incentive Cue

Paradoxically, research on general motivation suggests that the $\$ 1$ could in and of itself be a high-incentive cue. Specifically, Gray (1987) argued that when conditioned cues for a reward are encountered (e.g., conditioned monetary cues), people are more likely to display not only increased positive affects and appetitive motivational behavior directed toward a reward (e.g., cash), but also a heightened sensitivity to other reward cues in the environment. This finding suggests that providing a person with a monetary cue could increase the likelihood of the person indulging in other rewarding food items. What, then, are the circumstances in which receiving a monetary cue satiates the motivational state, and what are the circumstances in which the identical cue heightens the likelihood that a person will seek additional rewarding cues?

The purpose of this research is to address the boundary conditions of the findings in the constructs shown in the diagram above. While the authors found that mood states were not a factor in their results, I argue that how the mood was induced played a pivotal role in the results. As was demonstrated, receiving or not receiving the $\$ 1$ reward did not significantly alter a subject's mood, given that the person had a highincentive cue. However, $\$ 1$ also has a positive and measurable incentive value, and anyone receiving $\$ 1$ would feel good (i.e., the recipient could use it to buy something enjoyable).

\section{The Appraisal-Tendency Framework of Emotions}

The theory supporting my hypothesis draws upon the Appraisal-Tendency Framework (ATF) developed by Lerner and Keltner (2000). Briefly, ATF states that it is possible to experience conditions under which emotions of the same valence will have divergent effects. For example, it has been shown that anger triggered in one situation can evoke more optimistic risk estimates and risk-seeking choices in unrelated situations, whereas fear does the opposite: both fear and anger are described as states of feeling bad (Lerner, Gonzalez, Small \& Fischhoff, 2003; Lerner \& Keltner, 2001). This occurs because, first, each positive or negative emotion has its 
unique cognitive dimensions (Smith \& Ellsworth, 1985), and - depending on how each positive (or negative) emotion is coded - the likelihood of some specific course of action may vary (Lazarus, 1991; Scherer, 1999). Second, emotion carries with it certain motivational properties that spill over to subsequent judgments. Appendix A shows the constructs of ATF; Appendix B provides an example of the ATF approach to positive and negative emotions.

According to the ATF approach, positive emotions can be distinguished by two dimensions: Certainty and Responsibility. "Certainty" refers to the degree to which future events seem predictable versus unpredictable. "Responsibility" is the degree to which someone other than oneself seems responsible for the outcome. As shown in Appendix B, positive emotions of relatively high certainty are accompanied by relatively low responsibility (such as Pride) and vice versa (such as Surprise). In summary, receiving a small amount of cash for completing a task makes us feel good and is a predictable or anticipated reward, while receiving the same amount from a surprise windfall also makes us feel good, but the reward is unpredictable (Lerner \& Keltner, 2000; Smith \& Ellsworth, 1985). Therefore, given that we have a motivational drive a priori, the perception of an anticipated reward of $\mathrm{x}$ amount of dollars serves as the end goal of the drive. However, the latter perception that the possibility of receiving the same dollar reward is unpredictable serves as a motivation for some reward-seeking goal (which could be independent of any a priori motivational drive. In short: the cash reward serves different purposes, depending on whether it comes into one's possession as compensation or as a surprise.

Surprise is a short-lived emotion that can be conceptualized as a syndrome of reactions involving physiological changes (e.g., increased electro-dermal activity); behavioral changes (e.g., focusing of the attention and increased memory retention of the surprising stimulus); and a subjective feeling of surprise (Reisenzein, 2000; J Vanhamme, 2000). Surprise is elicited by a schema discrepancy - that is, it is elicited by unexpected products, services or attributes (Ekman \& Friesen, 1975; Scherer, 1984). The emotion of surprise has neither positive nor negative valence. Rather, it is the moderator of the subsequent elicited emotion (Charlesworth, 1969). For example, people who feel joy (or anger) after having been surprised will feel happier (or angrier) than if they had not previously been surprised.

Research into the role of surprise in marketing is still in the exploratory stages. To date, it has focused primarily on conjecturing the relationship between surprise and customer satisfaction. Preliminary support for the relationship between surprise and satisfaction has been shown in a series of studies by Oliver and Westbrook (Oliver, 
1993; Oliver \& Westbrook, 1993; Westbrook \& Oliver, 1991). In these studies, cluster analysis was used to group consumers according to the emotions they experienced during their purchase and consumption activities. The results demonstrated that a cluster characterized by strong emotions of surprise and joy also scored highest on the satisfaction scale. From a conceptual point of view, the intrinsic arousal of surprise might explain the relationship between surprise and satisfaction: the higher the satisfaction level, the higher the intrinsic level of arousal contained in the satisfaction response. Therefore, since surprise has a high potential for arousal, a surprised customer should feel a level of satisfaction with a high degree of arousal. In support of arousal as a key component in consumer satisfaction, Vanhamme and Lindgreen (2001) showed, through field studies using critical incident technique and diary analysis, that customer satisfaction is higher for positively surprising experiences than for comparable non-surprising experiences. Additionally, the more positively surprised consumers are by an experience, the higher their satisfaction level. However, the findings on surprise-behavior correlation are based on research observations made in an uncontrolled environment that was highly susceptible to confounding effects. To illustrate the validity of the construct, this research aims to investigate how positive emotions of varying arousal levels affect behavior in a controlled environment.

Among the studies that document the effects of specific emotions on subsequent behaviors, few have experimentally investigated the impact of distinct positive emotions (Cavanaugh, Bettman, Luce \& Payne, 2007). This gap is significant for three reasons. First, this research aims to show that a good feeling can serve as a means to achieve a goal rather than an end to the same goal fulfillment. ${ }^{3}$ Second, the field of behavioral economics (i.e., the application of psychological insights to economics) has been strongly influenced by cognitively focused research on decision-making but has remained largely untouched by researchers' recent interest in emotions, particularly positive emotions. The research here is intended to bridge this gap by providing a framework that can distinguish the wide range of discrete positive emotions that remain largely unresearched (Lerner et al., 2007b) .

\footnotetext{
${ }^{3}$ For example, (Fishbach, Shah, \& Kruglanski, 2004) focused on the affective transfer from separate goals (weight-watching vs. food enjoyment) to a singular means (eating). They showed that priming the goal to enjoy food led to eating hedonic food with more positive affect, whereas priming the goal of weight-watching imbued the same activity with negative affect. This line of study focuses on the resulting emotion evoked from performing a task (with a predictable outcome) and captures the possibility of incidental, unanticipated causes of positive emotion.
} 


\section{HYPOTHESIS DEVELOPMENT}

So, while (Wadhwa, et al., 2008) hypothesized that $\$ 1$ satiates the induced motivational state prior to a subsequent consumption task before the movie, this monetary reward attenuates the effects of reverse alliesthesia. I extend this more generally to:

H1a: Positive emotion which results from a predictable circumstance satiates the motivation to achieve a reward-seeking goal (such as being compensated $\$ x$ for some effort)

The constructs of $\mathrm{H} 1 \mathrm{a}$ are the same as those proposed in Experiment 2 by Wadhwa et al. (2008), which are shown in Figure 3 below:

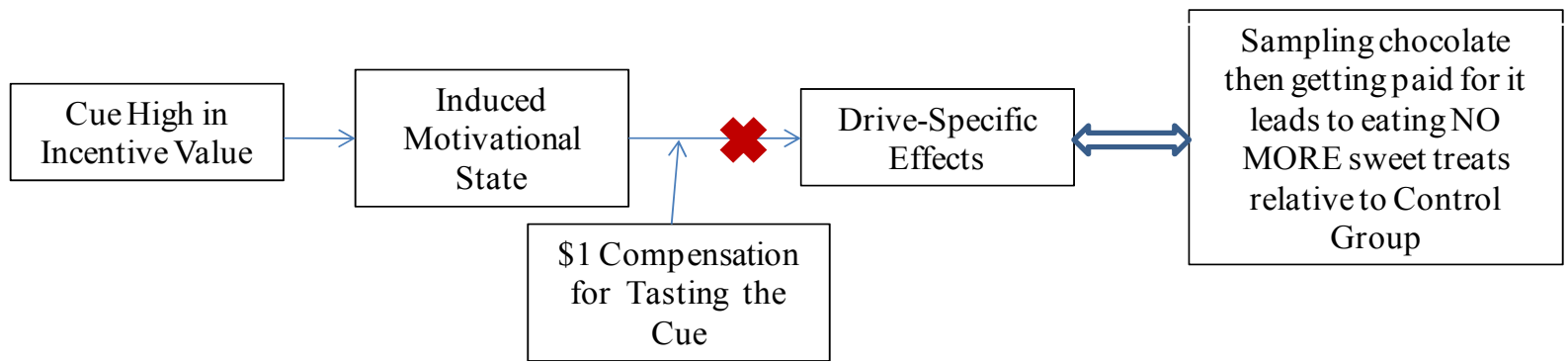

Figure 3 Satiation Of High Incentive Value Cue Induced Motivational State Via Monetary Compensation

Similarly, if the general motivational hypothesis holds, the order of compensation and the cue should be irrelevant, given that they have high incentives. That is, if the $\$ 1$ compensation is given first, it would be the cause of the induced general motivational state. This drive would then be satiated by sampling the expected high incentive cue as shown in Figure 4:

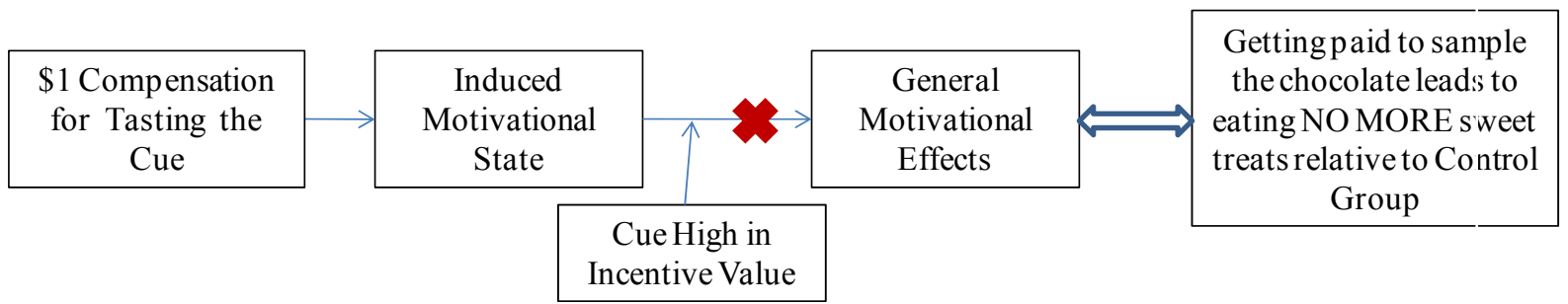

Figure 4 Satiation Of Monetary Compensation Induced Motivational State Via High Incentive Value Cue

H1b: Positive emotion which results from an unpredictable circumstance arising as a surprise serves as an incentive to motivate a reward-seeking goal (such as winning $\$ x$ from a lucky draw). See Figure 5. 


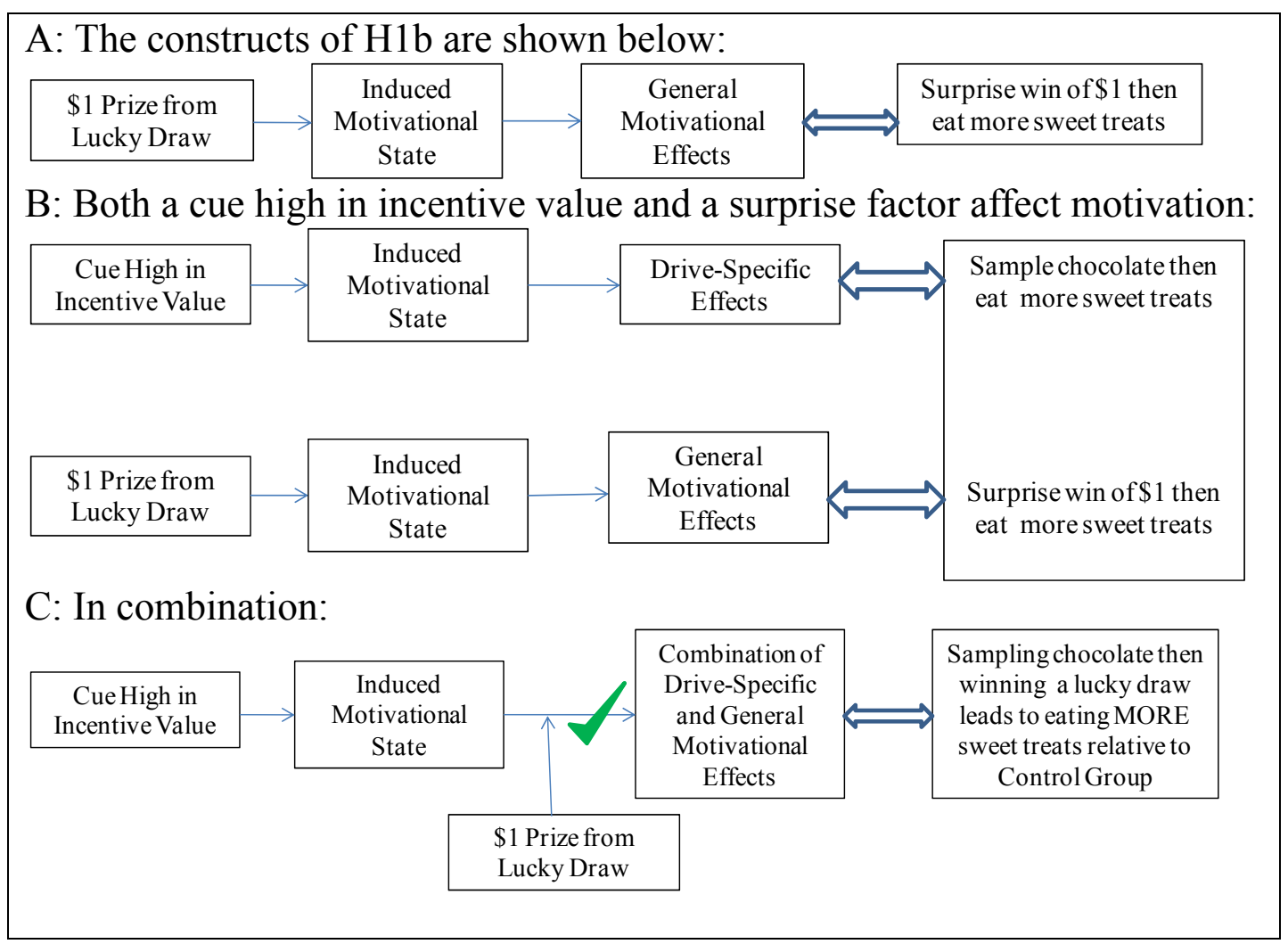

Figure 5 Summary Processes of Hypothesis $1 \mathrm{~b}$

Under the same logic, the induced motivational state could arise from winning a lucky draw, which could then increase the motivation to pursue rewarding goals. The subsequent and seemingly unrelated task of a sampling cue high in incentive value also independently leads drive-specific effects. This unrelated task is the means of motivating the pursuit of rewarding goals that already exist due to the surprise cash reward. We therefore expect to observe the manifestation of both drive-specific and general motivational effects on subsequent consumption behavior of rewarding items, as shown in Figure 6:

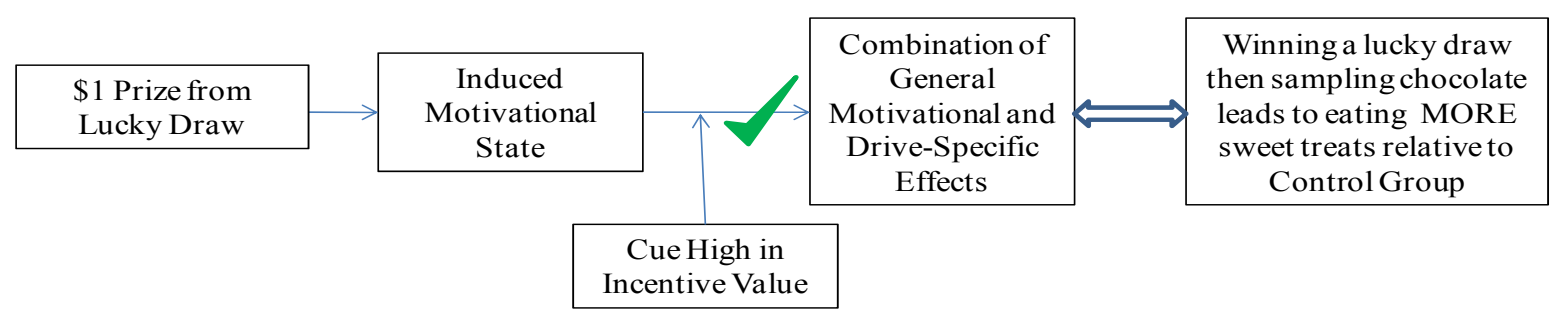

Figure 6 Generalizing Hypothesis $1 \mathrm{~b}$ 


\section{EXPERIMENT}

\section{Design and Procedure}

To test these hypotheses while remaining consistent with the literature on reverse alliesthesia, the experimental design was as follows: 2 (Positive emotion: predictable vs. unpredictable) x 2 (Induced emotion: pre- vs. post-sampling of high incentive cue) between subjects designed with an additional control condition. The study was presented in two seemingly unrelated phases. One hundred and thirty-eight undergraduates from three universities (mean age 22.4 years; 56.7\% female) were recruited from a large shopping mall located in northern Bangkok. The students were readily identifiable by their uniforms and were randomly assigned to one of the five conditions.

In Phase 1, half the non-control subjects were compensated with 50 Baht in cash (approximately the equivalent of US $\$ 1.60$ at the time) for participating in a market survey of a new chocolate product (a predictable condition). Half of the subjects in this group were immediately compensated upon arrival, while the other half were paid once they tasted and rated the chocolate product. The remaining non-control subjects received 50 Baht by winning a lucky draw ${ }^{4}$ (an unpredictable condition). Half the participants undergoing this unpredictable condition received the 50 Baht surprise win prior to sampling the chocolate product; the other half received the cash windfall after tasting and rating the chocolate. Every participant sampled one $20 \mathrm{~g}$ piece of high quality chocolate from Japan, which served as the new product to be rated. In this phase, the control group completed a filler task. Finally, the participants were thanked and then ushered into the next room.

Phase 2 was set up as a documentary film evaluation task. All of the participants were told that the movie would last about 15 minutes, and that free soft drinks (three leading brands of soft drinks) and snacks (seaweed and chocolate biscuits) would be provided courtesy of the movie producers. At the end of the movie, the calories consumed served as the dependent variable. After the movie, those in the manipulated conditions group were paid another 50 Baht each. Those in the control conditions group received 100 Baht. Finally, participants completed the 20-item PANAS mood scale (Watson, Clark \& Tellegen, 1988) and answered demographic questions before they were thanked and debriefed.

\footnotetext{
${ }^{4}$ The subjects were told that their group had been randomly selected to win a lump sum of cash to be shared among the group.
} 


\section{Manipulation Checks}

To verify that the experience was equally pleasant for everyone, a planned contrast on total affective state (PANAS) was carried out on the four groups, with manipulated conditions as well as the control condition group. Specifically, the null hypothesis was:

$\psi=4 * M_{\text {Control }}-M_{\text {Pre x Predictable }}-M_{\text {Post x Predictable }}-M_{\text {Pre x Unpreditable }}-M_{\text {Post x Unpreditable }}$

The null hypothesis was supported $t(134)=1.29$, NS.

However, as expected, those in the unpredictable condition group scored higher on the PANAS items related to unpredictability (Alert and Excited) relative to those with the predictable condition $\left(M_{\text {Predictable }}=4.82, \mathrm{SD}=.65 ; M_{\text {Unpredictable }}=4.05\right.$, $\mathrm{SD}=.84 ; F(1,134)=3.99, p<.05)$. In comparison, the unpredictable condition group scored lower on the PANAS items related to predictability (Proud and Strong) relative to those with the predictable condition $\left(M_{\text {Predictable }}=4.11, \mathrm{SD}=.65\right.$; $\left.M_{\text {Unpredictable }}=4.95, \mathrm{SD}=.72 ; F(1,134)=3.58, p<.05\right)$.

The above results indicate that snacking while watching a documentary is a pleasant experience. However, they also suggest that 50 Baht received for two different reasons (earned or windfall) induces different emotional states.

\section{RESULTS}

An ANOVA on the amount of total calories consumed showed a significant main effect on the unpredictable positive emotional (lump-sum cash won) condition ( $F(1$, $134)=3.58, p<.05)$; this effect was qualified by a significant type of emotion $\mathrm{x}$ order of receiving monetary reward interaction $(F(1,134)=3.84, p<.05)$. The amount of calories consumed was significantly higher for participants who received 50 Baht as a surprise windfall (i.e., those in the unpredictable positive emotional condition, in which $\mathrm{M}=187.25$ calories) than it was for both those who received 50 Baht framed as compensation (i.e., those in the predictable positive emotional condition; $\mathrm{M}=153.88$ calories), and those who were in the control condition group $(\mathrm{M}=148.67$ calories $)$.

The pattern of results supports the extant understanding of reverse alliesthesia: high-incentive cues activate a more general motivational state, prompting people to engage in reward-seeking behaviors, and such behaviors may be satiated prior to a subsequent consumption task. However, the pattern of results also shows that the same object, when perceived as a surprise, may bolster the effect of reverse alliesthesia. Specifically, those who first received the cash in an unpredictable manner before going on to sample the high-incentive value chocolate ended up consuming the 
greatest amount in terms of calories $(M=195.07$ calories and $M=179.43$ calories respectively). The results are shown in Table 1.

Table 1 Average Calories Of Hedonic Snacks Consumed

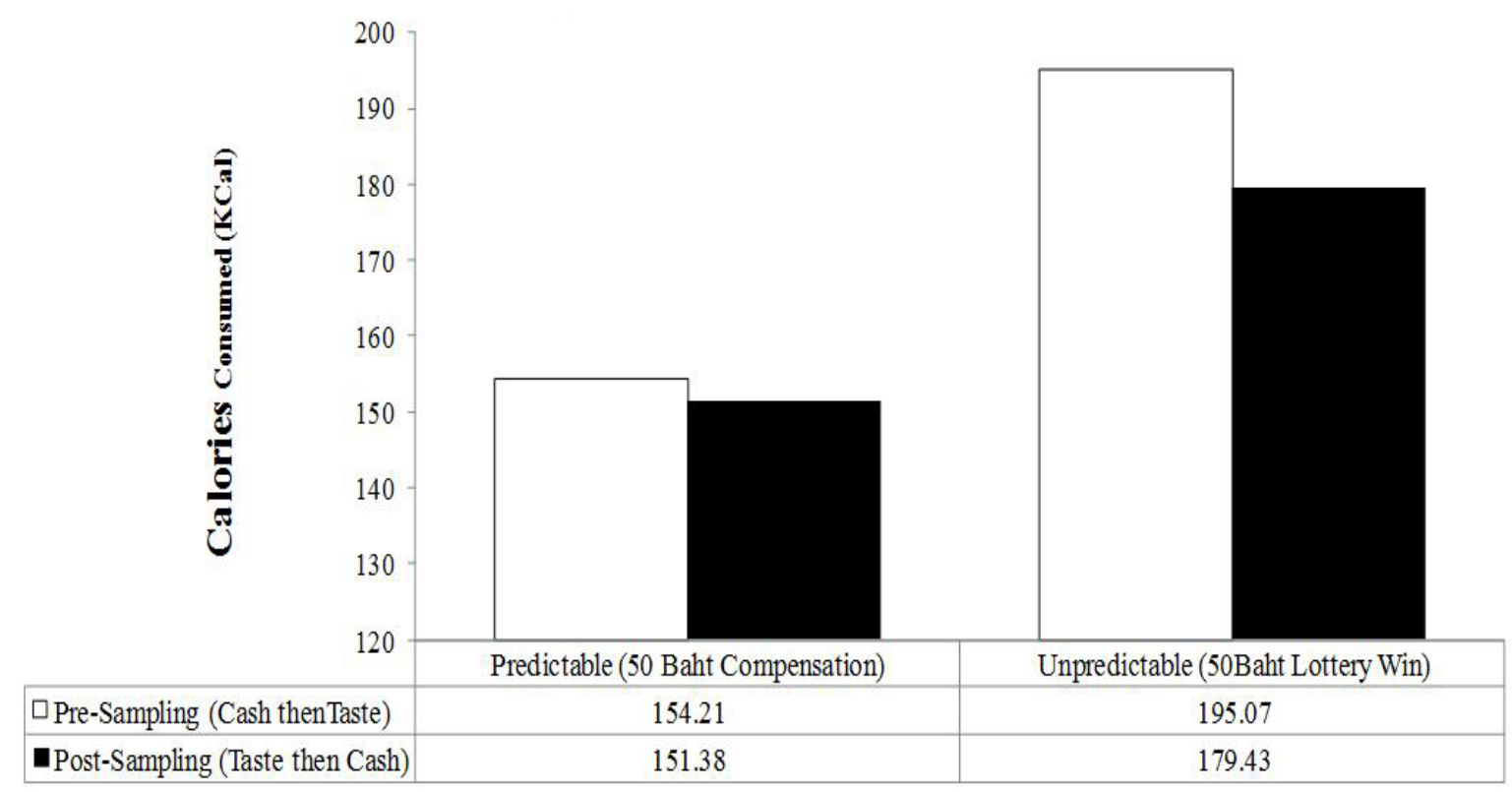

\section{DISCUSSION}

The pattern of reverse alliesthesia occurring at a general motivational level replicated that of Wadhwa et al. (2008). Additionally consistent with previous findings on satiation, we found that the effect of consumption cues high in incentive value on reward-seeking behavior to be attenuated when the activated motivational state was satiated by inducing predictable positive emotion with monetary compensation. Similarly, the general motivation which arose from receiving 50 Baht in compensation was also satiated by the consumption of the high-incentive cue of a chocolate treat. The results imply that in both instances, the latter emotional intervention was predictable and thus served as the end to the general motivational level drive for reward-seeking behavior (because receiving compensation and tasting chocolate are both rewarding experiences). In other words, satiation of the general motivational drive may be caused by various forms of high-incentive cues, as long as it is perceived as predictable.

The results demonstrated that the total caloric consumption of soft drinks and snacks for both of the predictable consumption groups was not statistically different from the baseline volume of the control group. In other words, being paid to sample something that tastes good satiated the prediction of reverse alliesthesia. The 
predictable post-group prediction is consistent with previous findings on reverse alliesthesia, while the predictable pre-group prediction adds to our understanding of the influence of incidental emotions and goal-seeking on reverse alliesthesia.

However, we found that inducing unpredictable positive emotion did not satiate the motivational drive which arose from sampling a chocolate treat. The drive remained unsatiated because of the unpredictability inherent in the surprise, which, according to the appraisal tendency framework, will be encoded as attributed not to oneself and therefore will not be used as an end goal to some prior reward-seeking behavior. Thus, the prediction for the unpredictable post-consumption group provides a boundary condition for the extant work on goal satiation in reverse alliesthesia; receiving a monetary reward may not always satiate a general motivational drive.

Although not predicted, there was a significant difference in the order in which the incentive cue was sampled. Those who first received the surprise money before sampling the chocolate went on to consume more hedonic foods than the group who sampled the chocolate before receiving the surprise cash award. This finding raises a question about the relationship between stimuli and hedonic dependent variables. Would the results be different if the hedonic dependent measures had a monetary value rather than a caloric content? We leave this possibility of unrelated motivational drive for future research.

\section{REFERENCES}

Aarts, H., Custers, R., and Marien, H. (2008). Preparing and motivating behavior $\begin{array}{llll}\text { outside of awareness. } & \text { Science, } & \text { 319(5870), } & 1639 .\end{array}$ http://dx.doi.org/10.1126/science.1150432.

Ariely, D. (2010). The Upside of Irrationality: The Unexpected Benefits of Defying Logic at Work and at Home. New York: HarperCollins.

Ariely, D., and Jones, S. (2008). Predictably Irrational: The Hidden Forces That Shape Our Decisions. New York: Harper.

Atkinson, J., and Birch, D. (1970). On the dynamics of action. Nederlands Tijdschrift voor de Psychologie en haar Grensgebieden, 25(2), 83.

Baumeister, R., Vohs, K., and Nathan DeWall, C. (2007). How emotion shapes behavior: Feedback, anticipation, and reflection, rather than direct causation. Personality and Social Psychology Review, 11(2), 167-203. http://dx.doi.org/10.1177/1088868307301033.

Berridge, K. (2000). Reward learning: Reinforcement, incentives, and expectations. Psychology of learning and motivation, 40, 223-278. 
http://dx.doi.org/10.1016/S0079-7421(00)80022-5.

Bodenhausen, G., Kramer, G., and Süsser, K. (1994). Happiness and stereotypic thinking in social judgment. Journal of Personality and Social Psychology, 66(4), 621-632. http://dx.doi.org/10.1037//0022-3514.66.4.621.

Cabanac, M. (1979). Sensory Pleasure. Quarterly Review of Biology, 54(1), 1-29. http://dx.doi.org/10.1086/410981.

Cavanaugh, L., Bettman, J., Luce, M., and Payne, J. (2007). Appraising the appraisaltendency framework. Journal of Consumer Psychology, 17(3), 169-173. http://dx.doi.org/10.1016/S1057-7408(07)70024-4.

Charlesworth, W. (1969). The role of surprise in cognitive development. Studies in cognitive development. Essays in honor of Jean Piaget, 257-314.

Cornell, C., Rodin, J., and Weingarten, H. (1989). Stimulus-induced eating when satiated. Physiology \& Behavior, 45(4), 695-704. http://dx.doi.org/10.1016/00319384(89)90281-3.

Custers, R., and Aarts, H. (2005). Positive affect as implicit motivator: On the nonconscious operation of behavioral goals. Journal of Personality and Social Psychology, 89(2), 129-142. http://dx.doi.org/10.1037/0022-3514.89.2.129.

Ekman, P., and Friesen, W. (1975). Unmasking the face: A guide to recognizing emotions from facial clues. Englewood Cliffs, NJ: Prentice-Hall.

Fishbach, A., Shah, J., and Kruglanski, A. (2004). Emotional transfer in goal systems. Journal of Experimental Social Psychology, 40(6), 723-738. http://dx.doi.org/10.1016/j.jesp.2004.04.001.

Forgas, J., and Bower, G. (1988). Affect in social judgments. Australian Journal of Psychology, 40(2), 125-145. http://dx.doi.org/10.1080/00049538808259077.

Forgas, J., and Locke, J. (2005). Affective influences on causal inferences: The effects of mood on attributions for positive and negative interpersonal episodes. Cognition and emotion, 19(7), 1071.

Gray, J. (1987). Perspectives on anxiety and impulsivity: A commentary. Journal of Research in Personality, 21(4), 493-509. http://dx.doi.org/10.1016/00926566(87)90036-5.

Han, S., Lerner, J., and Keltner, D. (2007). Feelings and consumer decision making: The appraisal-tendency framework. Journal of Consumer Psychology, 17(3), 158-168. http://dx.doi.org/10.1016/S1057-7408(07)70023-2.

Johnson, E. J., and Tversky, A. (1983). Affect, generalization, and the perception of risk. Journal of Personality and Social Psychology, 45(1), 20-31. http://dx.doi.org/10.1037//0022-3514.45.1.20. 
Kambouropoulos, N., and Staiger, P. (2001). The influence of sensitivity to reward on reactivity to alcohol-related cues. Addiction (on-Line), 96(8), 1175-1185. http://dx.doi.org/10.1046/j.1360-0443.2001.968117510.x.

Lazarus, R. (1991). Emotion and Adaptation. New York: Oxford.

Lerner, J., Gonzalez, R., Small, D., and Fischhoff, B. (2003). Effects of fear and anger on perceived risks of terrorism: A national field experiment. Psychological Science, 14(2), 144-150. http://dx.doi.org/10.1111/1467-9280.01433.

Lerner, J., Han, S., and Keltner, D. (2007a). Feelings and Consumer Decision Making: Extending the Appraisal-Tendency Framework. Journal of Consumer Psychology, 17(3), 181-187. http://dx.doi.org/10.1016/S1057-7408(07)70027-X.

Lerner, J., and Keltner, D. (2000). Beyond valence: Toward a model of emotionspecific influences on judgement and choice. Cognition \& Emotion, 14(4), 473493. http://dx.doi.org/10.1080/026999300402763.

Lerner, J., and Keltner, D. (2001). Fear, anger, and risk. Journal of Personality and Social Psychology, 81(1), 146-159. http://dx.doi.org/10.1037//00223514.81.1.146.

Lerner, J., Small, D., and Loewenstein, G. (2004). Heart strings and purse strings. Psychological Science, 15(5), 337-341. http://dx.doi.org/10.1111/j.09567976.2004.00679.x.

Loewenstein, G., and Lerner, J. (2003) The role of affect in decision making. In Davidson R, Goldsmith H, Scherer K Handbook of Affective Science (pp. 619642). Oxford: Oxford University Press.

Oliver, R. (1993). Cognitive, affective, and attribute bases of the satisfaction response. Journal of Consumer Research, 20(3), 418-430. http://dx.doi.org/10.1086/209358.

Oliver, R., and Westbrook, R. (1993). Profiles of consumer emotions and satisfaction in ownership and usage. Emotion, 6, 12-27.

Reisenzein, R. (2000). Exploring the strength of association between the components of emotion syndromes: The case of surprise. Cognition \& Emotion, 14(1), 1-38. http://dx.doi.org/10.1080/026999300378978.

Scherer, K. (1984). On the nature and function of emotion: A component process approach. Approaches to emotion, 293-317.

Scherer, K. R. (1999) Appraisal Theory. In Handbook of Cognition and Emotion (Eds T. Dalgleish and M. J. Power), United Kingdom: John Wiley \& Sons, Ltd. 
Schwarz, N., and Clore, G. (1983). Mood, misattribution, and judgments of wellbeing: Informative and directive functions of affective states. Journal of Personality and Social Psychology, 45(3), 513-523. http://dx.doi.org/10.1037//0022-3514.45.3.513.

Smith, C., and Ellsworth, P. (1985). Patterns of cognitive appraisal in emotion. Journal of Personality and Social Psychology, 48(4), 813-838. http://dx.doi.org/10.1037//0022-3514.48.4.813.

Toates, F. (1986). Motivational systems: Cambridge Univ Press.

Vanhamme, J. (2000). The link between surprise and satisfaction: An exploratory research on how best to measure surprise. Journal of Marketing Management, 16(6), 565-582. http://dx.doi.org/10.1362/026725700785045949.

Vanhamme, J., and Lindgreen, A. (2001). Surprise as a Marketing Tool? Paper presented at the Australian and New Zealand Marketing Academy Conference, Massey University, Wellington, New Zealand.

Wadhwa, M., Shiv, B., \& Nowlis, S. (2008). A bite to whet the reward appetite: The influence of sampling on reward-seeking behaviors. Journal of Marketing Research, 45(4), 403-413. http://dx.doi.org/10.1509/jmkr.45.4.403.

Watson, D., Clark, L. A., and Tellegen, A. (1988). Development and validation of brief measures of positive and negative affect: The PANAS scales. Journal of Personality and Social Psychology, 54(6), 1063-1070. http://dx.doi.org/10.1037//0022-3514.54.6.1063.

Westbrook, R., and Oliver, R. (1991). The dimensionality of consumption emotion patterns and consumer satisfaction. Journal of Consumer Research, 18(1), 84-91. http://dx.doi.org/10.1086/209243.

Wright, W. F., and Bower, G. H. (1992). Mood effects on subjective probability assessment. Organizational Behavior and Human Decision Processes, 52(2), 276-291. http://dx.doi.org/10.1016/0749-5978(92)90039-A. 


\section{Appendix A: Construct of Appraisal Tendency Framework}

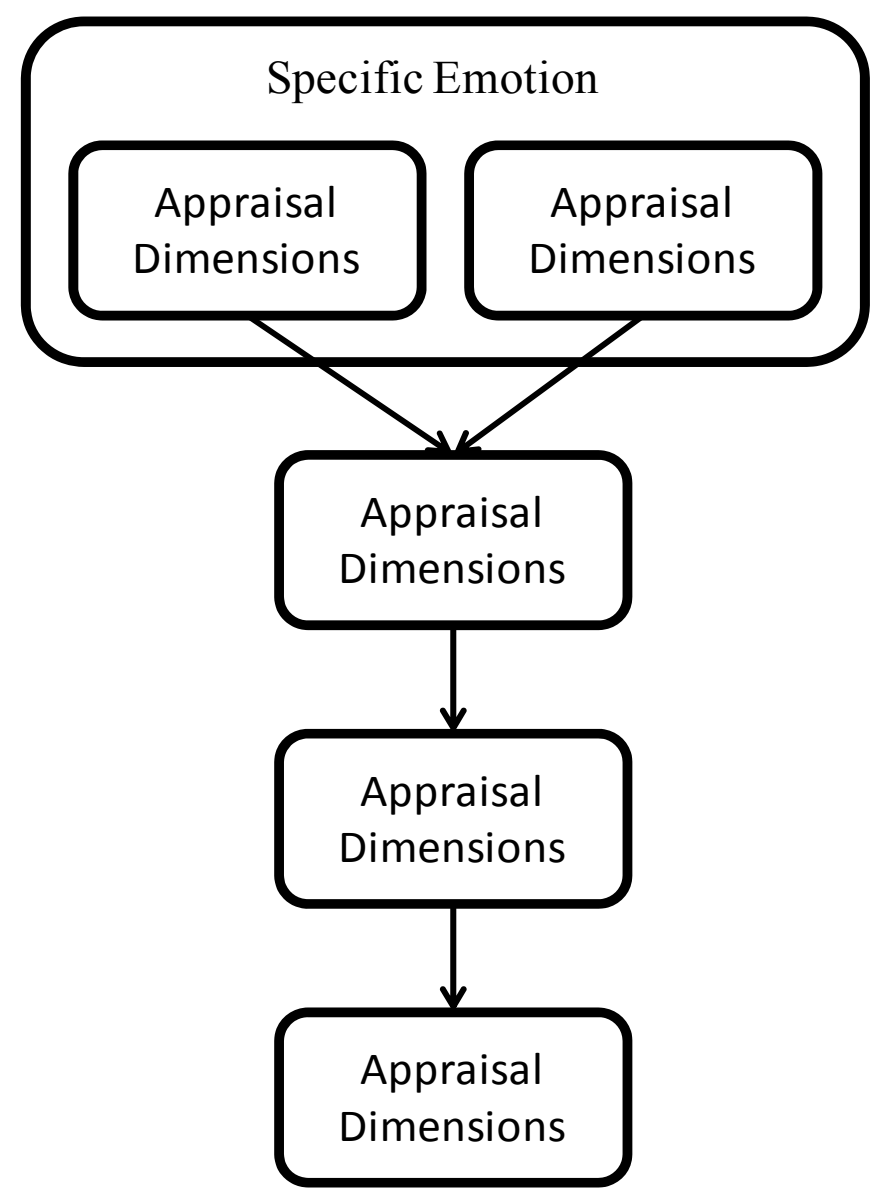


Appendix B: Illustrations of the ATF Approach (Lerner \& Keltner, 2000)

\begin{tabular}{|c|c|c|c|c|}
\hline & \multicolumn{2}{|c|}{ Illustration with positive emotions } & \multicolumn{2}{|c|}{ Illustration with negative emotions } \\
\hline & Pride & Surprise & Anger & Fear \\
\hline Certainty & Medium & Low & High & Low \\
\hline Pleasantness & High & High & Low & Low \\
\hline Attentional Activity & Medium & Medium & Medium & Medium \\
\hline Anticipated Effort & Medium & Medium & Medium & High \\
\hline Control & Medium & Medium & High & Low \\
\hline Responsibility & Low & High & High & Medium \\
\hline \multirow[t]{2}{*}{ Appraisal Tendency } & $\begin{array}{l}\text { Perceive positive } \\
\text { events as brought } \\
\text { about by self }\end{array}$ & $\begin{array}{l}\text { Perceive positive } \\
\text { events as unpredictable } \\
\& \text { brought about by } \\
\text { others }\end{array}$ & $\begin{array}{l}\text { Perceive negative } \\
\text { events as predictable, } \\
\text { under human control, } \\
\& \text { brought about by }\end{array}$ & $\begin{array}{l}\text { Perceive negative } \\
\text { events as unpredictable } \\
\& \text { under situational } \\
\text { control }\end{array}$ \\
\hline & \multicolumn{2}{|c|}{ Influence on attribution } & \multicolumn{2}{|c|}{ Influence on risk perception } \\
\hline $\begin{array}{l}\text { Influence on Relevant } \\
\text { Outcome }\end{array}$ & $\begin{array}{l}\text { Perceive self as } \\
\text { responsible }\end{array}$ & $\begin{array}{l}\text { Perceive others as } \\
\text { responsible }\end{array}$ & Perceive low risk & Perceive high risk \\
\hline
\end{tabular}

